Preprint typeset using LATEX style emulateapj v. 14/09/00

\title{
SPATIAL RESOLUTION OF HIGH-VELOCITY FILAMENTS IN THE NARROW-LINE REGION OF NGC 1068: ASSOCIATED ABSORBERS CAUGHT IN EMISSION?
}

\author{
GERALD CECIL \\ Dept. of Physics \& Astronomy, U. of N. Carolina, Chapel Hill, NC 27599-3255 \\ gerald@thececils.org \\ Michael A. Dopita, BRent Groves \\ Institute for Advanced Studies, Australian National University, Canberra ACT, Australia \\ ANDREW S. WILSON \\ Dept. of Astronomy, U. of Maryland, College Park, MD 20742 \\ Pierre Ferruit, Emmanuel PÉCONTAL \\ Observatoire de Lyon, France \\ AND \\ LUC BINETTE \\ UNAM, Mexico
}

\begin{abstract}
Using the STIS spectrograph on HST we have obtained a grid of [O III] $\lambda \lambda 4959,5007$ and $\mathrm{H} \beta$ emission-line spectra at $0 !^{\prime \prime} 05 \times 0 !^{\prime \prime} 19^{\prime \prime}$ and $60 \mathrm{~km} \mathrm{~s}^{-1}$ (FWHM) resolution that covers much of the NLR of NGC 1068. We find emitting knots that have blueshifted radial velocities up to $3200 \mathrm{~km} \mathrm{~s}^{-1}$ relative to galaxy systemic, are $70-150 \mathrm{pc}$ $\mathrm{NE}$ of the nucleus and up to $40 \mathrm{pc}$ from the radio jet, emit several percent of the NLR line flux but no significant continuum, span a small fraction of the sky as seen from the nucleus, coincide with a region of enhanced IR coronal-line emission, show gradients in radial velocities of up to $2000 \mathrm{~km} \mathrm{~s}^{-1}$ in $7 \mathrm{pc}$, span velocity extents averaged over $0 . \prime 1 \times 0 . \prime 2$ regions of up to $1250 \mathrm{~km} \mathrm{~s}^{-1}$, have ionization parameter $\mathcal{U} \gtrsim 0.1$, and ionized masses $\sim 200 \mathrm{M}_{\odot} / n_{e, 4}\left(n_{e, 4}=10^{4} \mathrm{~cm}^{-3}\right)$. The brightest parts of the blueshifted knots are often kinematically contiguous with more massive clouds nearer the jet that are moving with velocities of $\leq 1300 \mathrm{~km} \mathrm{~s}^{-1}$ relative to galaxy

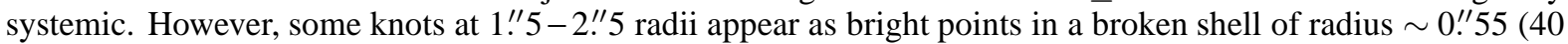

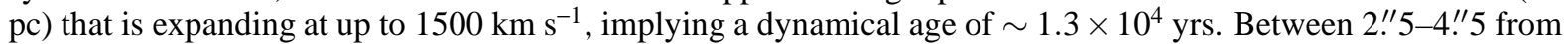
the nucleus, emission is redshifted relative to systemic, a pattern that we interpret as gas in the galaxy disk being pushed away from us by the NE radio lobe. We argue that the blueshifted knots are ablata from disintegrating molecular clouds that are being photoionized by the AGN, and are being accelerated radiatively by the AGN or mechanically by the radio jet. In their kinematic properties, the knots resemble the associated absorbers seen projected on the UV continua of some AGN.
\end{abstract}

Subject headings: Galaxies: Active — Galaxies: Individual (NGC 1068) — Galaxies: Nuclei — Galaxies: Jets — Galaxies: Kinematics and Dynamics — Galaxies: Seyfert

\section{INTRODUCTION}

Emission-line profiles that extend over several thousand $\mathrm{km} \mathrm{s}^{-1}$ are hallmarks of activity in galaxy nuclei of all luminosities. Some AGN also show absorption lines at UV rest wavelengths, originating from warm gas located outside the region that generates the nonstellar continuum. This gas is invariably blueshifted over a range of several thousand $\mathrm{km} \mathrm{s}^{-1}$ from the galaxy systemic velocity, yet often shows discrete components that each span several hundred $\mathrm{km} \mathrm{s}^{-1}$ (Hamann et al. 1997). What process accelerates the clouds?

Spatially complete spectral maps of nearby active galaxies can separate clouds that are agitated mechanically by the AGN from those that are simply illuminated by AGN photons (e.g. undisturbed gas in an "ionization cone"). In this paper we report on observations made with the Space Telescope Imaging Spectrograph (STIS) and its medium-resolution (M) gratings to map the "Narrow-Line Region" (NLR) of the Seyfert galaxy NGC 1068. In this NLR, Alloin et al. (1983) sampled a cloud complex that spans $\sim 200 \mathrm{pc}$, is centered $\sim 210 \mathrm{~km} \mathrm{~s}^{-1}$ blueward of galaxy systemic velocity, and has $\sim 1670 \mathrm{~km} \mathrm{~s}^{-1}$ FWHM ( $2200 \mathrm{~km} \mathrm{~s}^{-1}$ according to the HST FOS spectra of
Caganoff et al. (1991)) velocity extent. The complex of clouds discovered by Walker (1968), and mapped in detail by Cecil, Bland, \& Tully (1990, CBT hereafter) and Arribas, Mediavilla, \& Garcia-Lorenzo (1996) (see also Baldwin, Wilson, \& Whittle (1987)), was shown from FOS spectra (Kraemer, Ruiz, \& Crenshaw 1998) to be mostly photoionized by nuclear radiation, and to have bulk motion consistent with acceleration that is independent of radius (Crenshaw \& Kraemer 2000b, CKb hereafter). A recent STIS spectrum (Kraemer \& Crenshaw 2000, KC1 hereafter) shows that emission-line centroids near the "continuum hotspot" (a feature with substantial scattered nuclear light located $\sim 0$.' 17 [12 pc] $\mathrm{N}$ of the optically obscured nucleus) exhibit a trend towards greater blueshifted velocities as the ionization potential of the line species increases, a result that extends the earlier finding by Marconi et al. (1996) who found that coronal, near-IR lines are extended across a $\leq 4^{\prime \prime}$ region.

Section 2 describes the acquisition and reduction of the STIS spectra. We show in $\S 3$ that the fastest knots have been accelerated to radial velocities blueshifted from galaxy systemic by $>3200 \mathrm{~km} \mathrm{~s}^{-1}$, a pattern not evident in the single STIS spec- 
trum discussed by $\mathrm{KC} 1$ and $\mathrm{CKb}$. We consider various mechanisms capable of accelerating clouds and knots in $\S 4$, outline why the knots may be related to quasar "associated absorbers" in $\S 5$, briefly consider future observations to test this hypothesis in $\S 6$, and summarize our conclusions in $§ 7$. In this paper, we assume a distance to NGC 1068 of $14.4 \mathrm{Mpc}$, so $1^{\prime \prime}=70 \mathrm{pc}$.

\section{STIS LONG-SLIT SPECTRA}

\subsection{Data Acquisition}

Deep spectra at high spatial resolution are necessary to map and characterize the various dynamical subsystems in this NLR. We have therefore used the STIS, splitting $14 H S T$ orbits evenly between M-grating ( $\mathrm{R} \sim 5,000)$ maps of the [O III] and $\mathrm{H} \beta$ emission-line profiles, and less extensive $\mathrm{L}$-grating $(\mathrm{R} \sim 1,000)$ maps of far- and near-UV line fluxes. We used the 0 !' 2 -wide slit $(0 . \prime 19$ on sky) as a reasonable compromise between resolution and mapping efficiency. To maximize sensitivity, we binned pairs of pixels along the axis of wavelength dispersion during CCD readout of the M-grating spectra, yielding $60 \mathrm{~km} \mathrm{~s}^{-1}$ FWHM resolution.

We planned to obtain spectra both along and perpendicular to the radio jet. However, available guidestars restricted us to P.A. $38^{\circ}$, close to the axis of the large-scale radio jet. After a standard STIS peakup on the nucleus, we performed a smallangle offset perpendicular to the slit for each map position. We obtained five long-slit spectra before a safe event delayed the next spacecraft visit sufficiently that the large changes in the orbit of $H S T$ prevented us from reusing target offsets from guidestars. This required us to spend an unanticipated half-orbit a year later to reacquire guidestars. The added overhead, together with a slight positional shift between the two pointings, meant that we obtained a total of six spectra with parallel slits that cover $\sim 2 / 3$ of the area of the NLR, with spatial resolution $0 .{ }^{\prime \prime} 05$ along the slit (and jet axis) and $0 .{ }^{\prime \prime} 19$ in the perpendicular direction, see Fig. 1. We supplemented these data with several G430L and G430M spectra from the HST archive (P.I. $\mathrm{R}$. Antonucci) that were unbinned in wavelength and used the 0. .'1-wide slit; the three archival M-grating exposures yielded a single spectrum along P.A. $10^{\circ}$ that intersects the continuum hotspot, close to the P.A. $22^{\circ}$ orientation used by a published STIS spectrum (KC1). Standard STIS processing with on-thefly recalibration using optimal files produced wavelength and flux calibrated spectra whose parameters are listed in Table 1.

\subsection{Data Reduction}

We used the IRAF 'cosmic' task to remove charge spots and hot pixels. To derive line fluxes, we used the average reddening derived by $\mathrm{KC} 1$ for the blue-wing of the line profiles, $E_{\mathrm{B}-\mathrm{V}}=0.35$ as an initial guess to be refined at different points along the slits during modeling of our more dust-sensitive UV spectra (Groves et al. 2001). This reddening is consistent with estimates from the $[\mathrm{Fe}$ II $] \lambda 1.257 \mu \mathrm{m}$ to $1.64 \mu \mathrm{m}$ flux ratios across the NLR (Marconi et al. 1996).

The spectra were examined for instrumental scattered light following a procedure similar to that of Nelson et al. (2000); none was found. After masking emission lines, we used the IRAF 'continuum' task with third-order Chebyshev polynomials to model and subtract continuum light on a row by row basis.

From ground-based spectral maps (for example CBT, and Arribas, Mediavilla, \& Garcia-Lorenzo 1996), line emission in this NLR is known to be structured into roughly a halfdozen major subsystems with different kinematics, excita- tion, and geometric distribution. The gas distribution appears to be even more complex in the HST Faint Object Camera (FOC) image (Fig. 1), where many knots are unresolved spatially. This implies that there will be a "slit effect" on derived radial velocities, the magnitude of which depends on where the knot falls within our 0.' 19 -wide slit. This effect is small compared to the line widths of features in our data, and amount to $\pm 62 \mathrm{~km} \mathrm{~s}^{-1}$ ( \pm 1 resolution element) per maximum $\pm 0 .{ }^{\prime \prime} 095$ displacement (STIS Instrument Handbook, at http://www.stsci.edu/instruments/stis).

The lines of the $[\mathrm{O}$ III] $\lambda \lambda 4959,5007$ doublet are often blended because of the large range of gas velocities. To clean line profiles for analysis, we therefore used two complementary approaches: 1) parameterize each line with up to 25 Gaussians and constrain the two lines to have the correct theoretical ratio where they overlap, 2) multiply the profile of the red wing of [O III] $\lambda 5007$ by the theoretical $\lambda 4959 / 5007$ flux ratio and then subtract the result from the observed red wing of the $\lambda 4959$ line. We synthesized the [O III]-only image from our spectral grid, and then aligned this image to the FOC image to better than 0 .' 1 accuracy (half of the slitwidth); we thus verified that the FOC F509N filter passes all velocity components in our spectra. Capetti, Macchetto, \& Lattanzi (1997) have performed absolute astrometry and aligned the FOC image with VLA radio images, finding that radio feature $\mathrm{S} 1$ coincides with the warped maser disk, presumably the AGN, while $\mathrm{S} 2$ is a knot in the jet.

\section{EMPIRICAL RESULTS}

\subsection{Kinematic Components in the [O III] and $\mathrm{H} \beta$ Emission-Line Profiles}

\subsubsection{Overview}

Our STIS spectra resolve the spatio-kinematic structure of this NLR, subdividing the major complexes into three major kinematic components that have different velocity substructure yet similar [O III] and $\mathrm{H} \beta$ line profiles:

1. that associated with the rotating galaxy and its ionization cone (the narrow "spike" in the NE quadrant, see Alloin et al. 1983),

2. redshifted filaments, mostly associated with the NE radio lobe, which arise as the lobe pushes down on the disk, as originally suggested by Pécontal et al. (1997, PFBW hereafter),

3. blueshifted components that, at fainter levels, extend to velocities of more than $3000 \mathrm{~km} \mathrm{~s}^{-1}$ from systemic (larger than the $2950 \mathrm{~km} \mathrm{~s}^{-1}$ separation of the [O III] doublet).

We have associated kinematic components with individual clouds or cloud complexes located in the FOC image (Fig. 1). Slit numbers are indicated in Fig. 1 and also refer to panel numbers in the spectrograms of the seven slit positions Fig. 2. We focus on the various blueshifted components, summarizing their line profiles and the velocity dependences of their $[\mathrm{O} \mathrm{III}] / \mathrm{H} \beta$ flux ratios in Fig. 3. All velocities are quoted relative to the systemic value of the galaxy $\left(1148 \mathrm{~km} \mathrm{~s}^{-1}\right.$, Brinks et al. 1997). 


\subsubsection{Components HVO-HV7}

These features are isolated knots or diffuse edges of bright clouds in the FOC [O III] image, and are labelled 0-7 in Fig. 1. Their line profiles in the left columns of Fig. 3 show:

1. A component whose flux centroid redshifts from systemic velocity at knot 0 to $600 \mathrm{~km} \mathrm{~s}^{-1}$ at knot 7 . It has two FWHM values: in knots $0-3$ it is $300-400 \mathrm{~km} \mathrm{~s}^{-1}$, while in knots $4-7$ it is $900 \mathrm{~km} \mathrm{~s}^{-1}$. The descriptions that follow omit this component.

2. An extended blue wing out to $-3200 \mathrm{~km} \mathrm{~s}^{-1}$, whose behavior in the different gas complexes we now detail. We refer to these as "high velocity" features HV0-HV7.

Complex 0 is sampled by slits $1 \& 2$. In the FOC image, it is the diffuse, SE, boundary of the brighter, central NLR. Flux peaks near systemic velocity in the SW part of this complex and then extends to $-2700 \mathrm{~km} \mathrm{~s}^{-1}$ as the distance from the nucleus increases by $0 . / 2$ (14 pc).

Complex 1, centered 0.'5 (35 pc) NE of complex 0 , is a remarkable cluster of roughly a dozen unresolved knots in the FOC image. The knots subdivide into a comparable number of bright, blueshifted peaks in each of slits 1 to 3 , and we can usually match a knot to a velocity component because the knots are separated along the slit. The spectrum of the knot in slit 1 shows a diagonal feature, which is resolved into a series of spatially resolved steps in space-velocity that each span $\sim 400 \mathrm{~km} \mathrm{~s}^{-1}$, indicating an increase in gas velocity with position along the slit from $\sim-400 \mathrm{~km} \mathrm{~s}^{-1}$ to $\sim-2500 \mathrm{~km} \mathrm{~s}^{-1}$ over 0. .'5 (35 pc). In slits 2 and 3, two increases with nuclear distance occur: from -250 to $-1250 \mathrm{~km} \mathrm{~s}^{-1}$ then -1200 to $-3200 \mathrm{~km} \mathrm{~s}^{-1}$ over the 0 !' $4^{\prime}$ (28 pc) extent of the complex. Despite multiple discrete spatial components, the overall emission-line profile is smooth.

Knot 2 appears only in slit 4, where it is distributed between -1800 and $-300 \mathrm{~km} \mathrm{~s}^{-1}$. It is elongated slightly $\mathrm{N}-\mathrm{S}$ in the FOC image.

Knot 3 is a spatially diffuse knot in slit 4, with gas between -100 and $-2400 \mathrm{~km} \mathrm{~s}^{-1}$. The trend along slit 4, through knots 2 and 3 and then farther NE, is that of an incomplete "Doppler ellipsoid" centered at $-600 \mathrm{~km} \mathrm{~s}^{-1}$ and extending around that velocity by $\pm 1500 \mathrm{~km} \mathrm{~s}^{-1}$. In the FOC image (Fig. 1) it appears as an elliptical ring of diameter 0. . 55 centered $2 . ! 2$ from $\mathrm{S} 1$ ( $50 \mathrm{pc}$ ) delineated by a half-dozen faint wisps (see pink broken ellipse in Fig. 1). This apparently disintegrating structure therefore has a dynamical age of $\sim 1.3 \times 10^{4}$ yrs.

Complex 4 is included in slit 5 , has 3 or 4 unresolved knots in the FOC image, and lies along the edge of complex G. Velocity features are evident in slit 5, and extend in two adjacent bands, from -200 to $-2900 \mathrm{~km} \mathrm{~s}^{-1}$ and from -1200 (faint) to $<$ $-3000 \mathrm{~km} \mathrm{~s}^{-1}$.

Complex 5 forms the diffuse, NE boundary of clouds G and $\mathrm{H}$. We may just resolve it in slit 6 into a linear gradient from -200 to $-2300 \mathrm{~km} \mathrm{~s}^{-1}$ as nuclear distance increases.
Knots 6 \& 7 lie outside the area mapped by our six parallel spectra, but are intersected by the archival observation with slit along P.A. $10^{\circ}$. These knots resemble others in the FOC image, with knot 6 extending to $-1800 \mathrm{~km} \mathrm{~s}^{-1}$ and knot 7 extending to $-1600 \mathrm{~km} \mathrm{~s}^{-1}$.

\subsubsection{Clouds}

Our spectra in the right-hand columns in Fig. 3 include all the large clouds first delineated by Evans et al. (1991). Several clouds were spanned by CKa with their single L-grating slit, so the local continuum properties that those authors established are also mentioned in the following remarks.

A, included in slit 3, has a small peak centered at $500 \mathrm{~km} \mathrm{~s}^{-1}$, in addition to weaker emission that spans -1900 to +1000 $\mathrm{km} \mathrm{s}^{-1}$.

B, included in slit 4, extends from $<-2200$ to $>+2500$ $\mathrm{km} \mathrm{s}^{-1}$ (the velocity extent is uncertain because of the difficult continuum subtraction in our narrow spectral bandpass). The emission extends $\sim 0$.' 2 along the slit. The associated continuum is the "hotspot" discussed in detail by CKa that is probably scattered nuclear light.

C, included in slit 5, extends from -1400 to $+2000 \mathrm{~km} \mathrm{~s}^{-1}$. $\mathrm{CKa}$ found that most of its continuum is scattered nuclear light. Bicknell et al. (1998) propose that this cloud is being agitated by knot $\mathrm{C}$ in the radio jet, which Roy et al. (2001) argue is a shock standing in the jet, based on its low proper motion in multi-epoch radio VLBI images.

D is also included in slit 5. A faint unresolved component reaches $-1800 \mathrm{~km} \mathrm{~s}^{-1}$ and attaches to brighter, spatially extended emission that spans -500 to $+1800 \mathrm{~km} \mathrm{~s}^{-1}$. CKa found that half of its continuum is scattered nuclear light.

E, included in slit 6 , spans -1500 to $+1300 \mathrm{~km} \mathrm{~s}^{-1}$, but is brightest between -500 and $+100 \mathrm{~km} \mathrm{~s}^{-1}$.

F, included in slit 3, has two separate plumes. That nearer to the nucleus extends from systemic velocity to $-2700 \mathrm{~km} \mathrm{~s}^{-1}$, that farther extends from -400 to $+2000 \mathrm{~km} \mathrm{~s}^{-1}$.

G, included in slit 5, starts abruptly at $-200 \mathrm{~km} \mathrm{~s}^{-1}$ and extends to $+1400 \mathrm{~km} \mathrm{~s}^{-1}$. It emits strong [Fe VII] (PFBW). At lower spectral resolution, $\mathrm{PFBW}$ and $\mathrm{KC} 1$ both found a very large flux ratio $[\mathrm{O} \mathrm{III}] / \mathrm{H} \beta=22$ near peak emission. However, our high-resolution spectra isolates this component more effectively, reducing the summed flux ratio to $\sim 17$.

H is sampled by slit 6. Its profile resembles that of cloud G, but is skewed to brighter emission at blueshifts between -500 and $-1300 \mathrm{~km} \mathrm{~s}^{-1}$ vs. -250 to $-900 \mathrm{~km} \mathrm{~s}^{-1}$ for G. CKa found that half of its continuum is scattered nuclear light.

J \& K are covered by slits $4+5$ and 6 , respectively. They lie on straight lines drawn back to the nucleus from, respectively, clouds G and H. Axon et al. (1997) discovered that these features are double peaked, and we find that they are centered first 
at $+500 \mathrm{~km} \mathrm{~s}^{-1}$ then at $-700 \mathrm{~km} \mathrm{~s}^{-1}$ as distance from the nucleus increases.

I is the brightest line-emitting part of gas associated with the SW radio lobe. Line profiles are double-peaked, with the stronger peak redshifted (near $+700 \mathrm{~km} \mathrm{~s}^{-1}$ ) and sharp in slits 1-3, but more diffuse in slits 4-6. As the nuclear distance increases by $1^{\prime \prime}$, velocity centroids shift from +250 to +800 $\mathrm{km} \mathrm{s}^{-1}$ in slit 5 and from systemic to $-500 \mathrm{~km} \mathrm{~s}^{-1}$ in slit 6 .

\subsubsection{Clouds Associated with the NE Radio Lobe}

Our slits sample many filaments across the base of this extended bow shock (Wilson \& Ulvestad 1987) in the interval 2$4^{\prime \prime}$ from the nucleus. Some filaments beyond clouds $\mathrm{G}$ and $\mathrm{H}$ and a few fainter knots beyond the top of Fig. 1 are blueshifted, but beginning at 2.' 6 radius most of what appears near the top of the FOC image as a coherent V-shaped feature is redshifted in slits 1-6, confirming the findings of PFBW. Between 2.'9 and 4 " radius, it is striking that individual components are resolved along the slit yet all range in velocity from systemic to +1200 $\mathrm{km} \mathrm{s}^{-1}$. There is a narrow feature near systemic velocity and only faint, diffuse emission to the blue. From the [S II] doublet in this region, PFBW derived gas densities near systemic velocity of $\sim 400 \mathrm{~cm}^{-3}$ if $T_{e}=10^{4} \mathrm{~K}$.

\subsection{Physical Properties}

We have used the line profiles displayed in Fig. 3 to constrain the ionized gas mass, kinetic energy, and momentum of each velocity component. A mask was used to isolate the component in the higher signal/noise ratio [O III] composite profile. This mask was then applied to the same velocity range in the dereddened $\mathrm{H} \beta$ profile. The resulting flux has been converted into an ionized gas mass, assuming case-B recombination conditions (for which $\mathrm{KC} 1$ found evidence in the red-wing component $\mathrm{NE}$ of the nucleus) and a pure $\mathrm{H}$ plasma. This conversion depends on the average electron density. In Table 2 we have therefore scaled some quantities by density $n_{e, 4}=10^{4} \mathrm{~cm}^{-3}$, which Dopita (1997) found was appropriate for the NLR. Undoubtedly, a large range of gas densities are present in each emitting feature.

The high-velocity features HV0-HV7 emit a few percent of the total NLR $\mathrm{H} \beta$ flux. In Table 2 we also tabulate properties of clouds A-H that were identified by Evans et al. (1991), and introduce clouds $\mathrm{J}$ and $\mathrm{K}$ (cloud I is rather ill-defined, in the SW radio lobe).

\section{DISCUSSION}

While the clouds might have been as blended at HST resolution as they are from the ground, in $\S 3$ we showed that our deep spectra have resolved spatially the NLR. We have also resolved much of it kinematically, although not down to the thermal width $\left(17 \mathrm{~km} \mathrm{~s}^{-1}\right.$ in $10^{4} \mathrm{~K}$ gas $)$ in part because of inadequate spectral resolution and in part because several components are still projected along each line of sight and may have turbulent substructure.

To track the energy flow and to isolate possible shock-excited structures, we must now constrain the internal properties and space velocities of the NLR clouds.

\subsection{Photoionization of the NLR Clouds}

The NLR clouds are bright features embedded in the largescale ionization cones and are distributed over several arcseconds, so have been studied extensively from space and, in aggregate, from the ground. FOS spectroscopy lacked spatial resolution to separate clouds from their surroundings. Subsequently, KC obtained a STIS spectrum along one cut through the NLR using low-resolution gratings that spans $\lambda \lambda 115-1027$ $\mathrm{nm}$. Along the slit, they analyzed continuum fluxes (CKa), line fluxes (KC1), and gas kinematics $(\mathrm{CKb})$. In $\mathrm{KC} 1$ they reproduced the observed flux ratios with AGN photoionization models that require considerable absorption along sight-lines from the nucleus that intersect the galaxy gas disk. They confirmed a correlation between ionization potential and line blueshift from galaxy systemic velocity that Marconi et al. (1996) had discovered in their ground-based IR spectra.

KC1 sampled several clouds NE of the nucleus, so their photoionization models also address the new features that we have mapped. Their models combine matter- and ionizationbounded clouds, the former arising plausibly from low-density, photoevaporated envelopes of the latter (Binette, Wilson, \& Storchi-Bergmann 1996). Matter-bounded clouds may filter AGN photons that impinge on the ionization-bounded cores, so internal dust would play a critical role in setting their emissionline flux ratios (Dopita et al. 2001; Groves et al. 2001).

The models of $\mathrm{KC} 1$ required that the blue-wing clouds be optically thin above the Lyman limit, and need another photon source between $1 . " 4-1 .{ }^{\prime \prime} 8$ to explain strong NUV lines and a larger He II $\lambda 4686 / \mathrm{H} \beta$ ratio along $202^{\circ}$ P.A. CKb suggested that clouds are decelerating here, forming a $\sim 1000 \mathrm{~km} \mathrm{~s}^{-1}$ shock that would brighten high-ionization lines as observed. They lacked the sensitivity and spatial coverage of Marconi et al. (1996) to coronal-line emission, so unlike those authors found no such emission beyond cloud B and the UV continuum hotspot. In $\mathrm{CKa}$ they established that the scatterer is tenuous and coincides with the deceleration region. They found reasonable fits to blue-wing line fluxes if $85-90 \%$ of the flux comes from tenuous gas with $\left(\log \mathcal{U}, n_{H}, \log N_{H}\right)=(-1.45 \pm 0.15,3 \times$ $\left.10^{3} \mathrm{~cm}^{-3}, 21.18 \pm 0.18 \mathrm{~cm}^{-2}\right)$ and the rest from denser clouds with $\left(\log \mathcal{U}, n_{H}, \log N_{H}\right)=\left(-2.9,2 \times 10^{4} \mathrm{~cm}^{-3}, 20.7 \pm 0.1 \mathrm{~cm}^{-2}\right)$, both components needing dust fractions of 10-25\% (larger values closer to nucleus) to suppress Ly $\alpha$ and C IV to their observed dereddened fluxes.

$\mathrm{KC} 1$ found that the red-wing clouds have the higher dust fraction $(25 \%)$ and are exposed to a lower ionizing flux (especially above the He II Lyman limit), indicating absorption of AGN photons on the way to the red wing gas, and thence by the tenuous blue-wing component along our line of sight. Marconi et al. (1996) find IR coronal lines across the inner $4^{\prime \prime}$ diameter that emit out to $2000 \mathrm{~km} \mathrm{~s}^{-1}$ blueshift from systemic velocity, and which can arise either in gas photoionized by a hard UV continuum or in a hot $\left(\sim 10^{6} \mathrm{~K}\right)$ collisionally ionized (perhaps shocked) plasma. Oliva et al. (2001) note that the [Fe II] emission is unlikely to come from shocks because they find that the flux ratio $[\mathrm{Fe} \mathrm{II}] /[\mathrm{P} \mathrm{II}] \sim 1,5 \%$ of the value seen in supernova remnants.

X-ray results also support photoionization NE of the nucleus, beyond radius $r_{i}$. First, Young et al. (2001) show that filaments in the Chandra ACIS image correlate well with those in $[\mathrm{O}$ III $] \lambda 5007$ and $\mathrm{H} \alpha+[\mathrm{N}$ II] images. Second, the Newton/XMM RGS spectrum reported by Paerels et al (2000) (also Behar et al. 2001) show that gas in this region is photoionized, based on the detection of narrow radiative recombination continua, line flux ratios within the helium-like triplets, and the weakness of Fe L-shell compared to K-shell emissions. 


\subsection{Deprojected Velocity Field}

Ground-based Fabry-Perot (CBT) and integral-field spectra (PFBW) have constrained the bulk motion and space distribution of the NLR clouds. CBT found an overall distribution of clouds in a thick bicone with maximum half opening angle $\sim 41^{\circ}$ and whose axis is inclined $\sim 85^{\circ}$ to our line of sight; PFBW showed that gas coincident with the NE radio lobe beyond 2." 5 radius is predominantly redshifted. $\mathrm{CKb}$ also posited a biconical flow to match the velocity centroids along their single STIS slit, requiring outflow velocities that reach 1300

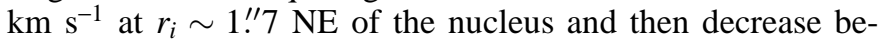
yond. Limited spatial coverage of a structure whose symmetry axis lies near the plane of the sky meant that their kinematical data are consistent with either an outflow that is radial from the nucleus or one that is roughly perpendicular to the jet axis. The archival M-grating spectrum (Fig. 4) shows that knots HV6 and 7 are comparable to the largest blueshifts in the model geometry of $\mathrm{CKb}$.

We see abrupt jumps in velocity as the STIS slits cross the clouds and knots evident in the FOC narrow-band image. In Fig. 5, we compare our line profiles with the projections of several models of radial and cylindrical outflow. By increasing the half angle of the NE bicone from $\sim 40$ to $50^{\circ}$ within the radius $r_{i}$ of the $\mathrm{CKb}$ model where outflow velocities begin to decrease, we better match the trend in the centroids of radial velocities of the blueshifted features in our spectra. The velocity limits of our two models are shown in the Figure, and correspond to: (solid line) increasing the maximum outflow to at least 2500 $\mathrm{km} \mathrm{s}^{-1}$ at $r_{i}$, and (long dashed line) having the gas expand from the jet axis with all of its motion along our line of sight. We also plot the outflow model of $\mathrm{CKb}$ in short dashed lines. The gas becomes increasingly blueshifted (on average) out to radius $2^{\prime \prime}$, implying its acceleration on that scale. However, the observed gas does not fill all of the NE bicone, because redshifted emission is observed at much lower velocities than predicted by all of the outflow models until the NE radio lobe is reached. Emission then jumps to a range that extends from zero to $>1000$ $\mathrm{km} \mathrm{s}^{-1}$ redshift (relative to systemic velocity) and persists from $3^{\prime \prime}$ to the middle of the NE radio lobe (which occurs at the top of the panels in Fig. 2); the emitting features are spatially compact. $\mathrm{KC} 1$ noted that the emission-line flux ratios of the red wing gas are consistent with irradiation by a heavily absorbed component. If these clouds are accelerated radiatively, they may not attain velocities as high as those that were irradiated directly.

As suggested by Tecza, Thatte, \& Maiolino (2001) based on infrared [Fe II] spectra and PFBW based on spatially deconvolved optical spectra, the red wing gas beyond $2 . " 5$ radius is apparently being pushed into the dense galactic disk by the lateral expansion of the NE radio lobe. Our favored scenario is shown in Fig. 6. The jet is inclined to the disk. Eventually, at radius $2^{\prime \prime}$, it bursts out of the disk and inflates the radio lobe. The lobe expands laterally in all directions, but is only visible where it pushes into the disk and creates the zero to redshifted emission. The large blueshifted extensions on the high-velocity clouds suggest that the clouds are moving slower than the HV knots. The HV flow must have been accelerated relative to the clouds or reoriented closer to our line of sight by a combination of bouyancy, radiative acceleration, and lobe/cloud collisions (Taylor, Dyson, \& Axon 1992). Depending upon their dynamics, the larger clouds may lag the knots because of larger drag forces, as $\mathrm{KC} 1$ have proposed for the red wing component.

\subsection{Acceleration of Clouds \& Knots}

\subsubsection{Jet Interactions with Clouds $C \& D$}

Using the long-slit mode of the FOC, Axon et al. (1997) found that line profiles are double-peaked and have highest gaseous excitation across the radio jet near clouds $\mathrm{G}$ and what we call K. On this basis they argued that hot shocked gas in the jet cocoon expands clouds from the jet axis. Nearby, Kraemer, Ruiz, \& Crenshaw (1998) found evidence from emissionline ratios in an FOS spectrum for cosmic-ray heating of the emission-line gas. The Merlin and VLA images of Gallimore et al. (1996, G96 hereafter), when registered using the astrometry of Capetti, Macchetto, \& Lattanzi (1997) (see red contours in Fig. 1), place NLR clouds B, C, D, and F adjacent to the radio jet; in particular, clouds $\mathrm{B}$ and $\mathrm{C}$ straddle a stationary radio knot (Roy et al. 2001) where the jet bends $\sim 23^{\circ}$. Bicknell et al. (1998) have modeled this complex as a jet-cloud interaction. What constraints do our data impose?

$\mathrm{KC} 1$ found that cloud $\mathrm{C}$ is photoionized. However, decomposing its profile in Fig. 3 into Gaussians isolates a component centered $\sim 300 \mathrm{~km} \mathrm{~s}^{-1}$ blueward of systemic and a wing component centered $300 \mathrm{~km} \mathrm{~s}^{-1}$ to the red, both with dispersions $\sim 500 \mathrm{~km} \mathrm{~s}^{-1}$. The blue component has flux ratio $[\mathrm{O} \mathrm{III]} \lambda 5007 / \mathrm{H} \beta \sim 16 v s . \sim 8-10$ for the red component. While the considerable line widths are consistent with shocks, the flux ratios are consistent with photoionization by the AGN with the red wing gas more absorbed. Thus, deflection of the jet at cloud $\mathrm{C}$ is not clearly connected to the excitation of the cloud. Cloud E, which is farther from the jet than cloud C, shows a similar blue feature but not one in its red wing.

The NE component of the nuclear radio triple is $0 . / 75 \mathrm{NE}$ of knot S1 (the nucleus). G96 note that this jet component, like the radio knot near cloud $\mathrm{D}$, has a flat synchrotron spectrum and extended emission that is misaligned with the jet. However, the jet does not bend at the NE component nor is there maser emission, arguing against a shock front. It is therefore interesting that the emission-line profiles of cloud $\mathrm{D}$ are double peaked. The blue peak has flux ratio $[\mathrm{O} \mathrm{III}] / \mathrm{H} \beta \sim 15$, but now the red peak ratio decreases monotonically through the profile from 17 to $<3$ over a $1000 \mathrm{~km} \mathrm{~s}^{-1}$ range. This decline implies that gas density increases with the velocity deviation from galaxy systemic, not what would be expected if most of the cloud mass is at velocities close to rest in the galaxy. G96 note that a cloud interacting with the jet must be sufficiently massive to avoid acceleration, implying

$$
\begin{aligned}
M_{\text {cloud } B} & \geq 40 M_{\odot}\left(\frac{r_{j}}{7.5 p c}\right)^{2}\left(\frac{\rho_{j}}{5 \times 10^{-27} \mathrm{gcm}^{-3}}\right) \times \\
& \left(\frac{v_{j}}{15,000 \mathrm{~km} \mathrm{~s}^{-1}}\right)\left(\frac{t_{\text {lobe }}}{3.5 \times 10^{5} y r}\right) .
\end{aligned}
$$

Here jet density and velocity are normalized to the values appropriate for the lighter jet model of Wilson \& Ulvestad (1987) for the NE radio lobe, $t_{\text {lobe }}$ is the age of the NE radio lobe from spectral aging as estimated by G96, and the jet radius $r_{j}$ was measured by Gallimore et al. (1994). If the average NLR cloud density is $\sim 150 \mathrm{~cm}^{-3}$, the entries in Table 2 show that clouds are massive enough to remain adjacent to the deflecting jet. This value is consistent with the mean densities of Galactic molecular clouds (Solomon et al. 1987).

Elsewhere, radio and line emission are uncorrelated. G96 note that this is expected if the jet has swept all gas from the region. The high-velocity knots might then be ablated from 
molecular clouds in the galaxy disk that have rotated into the jet. The clouds accelerated would all be blueshifted because they would move predominantly away from and above the galaxy disk. Similar clumps below the jet would sink into the denser disk where they would be subject to stronger drag forces and hence decelerated more effectively (consistent with the gas distribution in the spectral maps of PFBW).

\subsubsection{Shocks Near the X-ray Subpeak?}

Three characteristics of the XMM/Newton X-ray spectrum of NGC 1068 within $20^{\prime \prime}$ of the nucleus indicate that the gas is predominantly photoionized: (i) narrow radiative recombination continua; (ii) in the He-like triplets the forbidden lines are stronger than the resonance lines; and (iii) Fe $\mathrm{L}$ emission is weak compared to the $\mathrm{K}$ shell emission of the abundant elements (Kinkhabwala et al. 2001; Behar et al. 2001). However, subtraction of the predictions of a photoionization model from the spectrum shows residuals that may indicate a collisionally excited component or other effects (Paerels et al 2000).

Moreover, near the V-shaped line-emitting feature whose apex is $3^{\prime \prime} \mathrm{NE}$ of the nucleus (at top of Fig. 1), the 4-26 spectrum from the Chandra HETGS (Ogle et al. 2001) shows that resonance lines are brighter than forbidden lines, implying either a collisionally ionized component or strong resonant scattering. Ogle et al. (2001) show that the collisional component lies at the X-ray subpeak reported by Young et al. (2001). The X-ray subpeak may represent gas in the galactic disk on the far side of the NE lobe and being compressed by it (cf. PFBW). As discussed in $\S 4.1$, photoionization models of the UV and optical STIS spectra of KC1 also support this additional power source near the subpeak.

\subsubsection{Shocks Near Clouds $G-K$ ?}

Two lines of evidence argue for shocks near clouds G-K:

1. Clouds $\mathrm{G}$ and $\mathrm{K}$ are close to the "NE radio subpeak", located 2!' 1 NE of the obscured nucleus (Wilson \& Ulvestad 1987), suggesting dissipation of kinetic energy and possibly cloud acceleration by the radio jet.

2. Nearby we find the Doppler ellipsoid described in $\S 3.1 .2$, whose expansion velocity of $1500 \mathrm{~km} \mathrm{~s}^{-1}$ will generate a post-shock temperature of $\sim 3 \times 10^{7} \mathrm{~K}$ if the ambient medium is relatively stationary, fully ionized, and has $10 \%$ He abundance.

To estimate the mechanical luminosity of the Doppler ellipsoid, we assume that energy is injected at constant rate, that the shell shock is strong so that we can ignore the thermal pressure of the ambient gas, and that the bubble is within the scale height of the galaxy disk so that the ambient ISM density is constant. Then in the energy conserving phase of the bubble interior, the bubble radius and expansion velocity are related: $R=1.67 \mathrm{vt}$. By observation, $t=0.012$ Myr. Mechanical luminosity $L$ follows from $R(t)=31\left(L_{36} / n_{0}\right)^{0.2} t_{6}^{0.6} \mathrm{pc}=100 \mathrm{pc}$ with $t_{6}=0.012$ (where $L_{36}$ has units $10^{36} \mathrm{ergs} \mathrm{s}^{-1}$ and $n_{0}$ is the density of the ambient ISM in $\mathrm{cm}^{-3}$ ), so $L=5.2 \times 10^{7} n_{0} \mathrm{~L}_{\odot}$. Overall, the filaments that delineate the bubble and maybe its associated ISM shock emit $<2 \%$ of the NLR [O III] and $\mathrm{H} \beta$ fluxes.

\subsubsection{Radiative Acceleration of Dusty Clouds}

Dust absorption dominates opacity in a photoionized plasma like this NLR when ionization parameter $\mathcal{U}>\alpha\left(T_{e}\right) / c \kappa$, where $c$ is lightspeed, $\kappa \sim 10^{-21} \mathrm{~cm}^{2}$ atom ${ }^{-1}$ is the opacity assuming the standard Galactic reddening curve, and $\alpha\left(T_{e}\right) \sim 2 \times 10^{-13}$ is the recombination coefficient. The dust will compete successfully for ionizing photons in the highly ionized zone, suppressing line flux. A strong pressure gradient will also be set up so that the plasma density will be much enhanced by the time lower ionization species emit. In consequence, a lower $\mathcal{U} \sim 0.007$ will provide an apparent model fit to the spectrum, close to the value preferred by $\mathrm{KC}$ for their denser component. This ionization parameter is closely related to the value of $\mathcal{U}$ at which radiation pressure starts to dominate either the pressure gradient, or the dynamical acceleration of the ionized plasma. Thus, the flow of dusty clouds falling toward the nucleus is intrinsically unstable above the dust sublimation point, with clouds being driven into outflow in the direction of slowest accretion (i.e. within the ionization cones). Dopita et al. (2001) detail this scenario. In hydrodynamical simulations of cloud acceleration (Schiano, Christiansen, \& Knerr 1995), tenuous gas streams ablate from surfaces of massive clouds, which may be the source of the high-velocity features we observe.

In this NLR, the flux in the ionizing continuum needed to maintain the observed line luminosity $\left(\sim 3 \times 10^{44} \mathrm{ergs} \mathrm{s}^{-1}\right.$, $\mathrm{KC} 1)$ is comparable to the FIR luminosity $1.5 \times 10^{11} \mathrm{~L}_{\odot}$. Dusty clouds are accelerated radiatively by $\sim 10^{-5} \mathrm{~cm} \mathrm{~s}^{-2}$, so can reach their observed mean velocities of $\sim 500 \mathrm{~km} \mathrm{~s}^{-1}$ over 35 pc. In Fig. 3, the cloud line profiles indeed show less velocity structure than do the knots.

This scenario ignores the role of the radio jet in agitating the NLR clouds (Bicknell et al. 1998). But, as discussed earlier, its influence may be localized to line broadening and ablation in specific jet/cloud and lobe/cloud interactions, for example between B and C (Gallimore et al. 1996) and G and H (Axon et al. 1997). While the region of collisional line emission coincides with the base of the NE radio lobe, numerical simulations of a breakout lobe do not show (D. Balsara, private communication) a strong backflow vortex at its base that might form high-velocity shocks. In any event, many of the high-velocity knots have similar line profiles, and are found $>40 \mathrm{pc}$ from the brighter parts of the jet.

\section{ARE THE HIGH-VELOCITY KNOTS "ASSOCIATED ABSORBERS" SEEN IN EMISSION?}

If the HV emission knots in NGC 1068 were instead viewed against the nuclear continuum, they would resemble in their kinematics the Associated Absorption Line (AAL) systems seen in a few percent of quasars (Hamann et al. 1997, for example). $\mathrm{KC} 1$ argue from their spectral models that the putative UV absorber in the NE quadrant must block less than half of the nuclear light. If the blueshifted knots have the same extent along the line of sight as we see on the sky (assumed here to be just resolved), they would cover at most $2 \%$ of the sky at the nucleus, consistent with values derived for some AAL absorbers.

Such absorbers are usually thought to lie much closer to the source of hard photons than the 35-160 pc radial distance of the clumps in NGC 1068. However, recent photoionization models of high-resolution quasar spectra are establishing that AAL absorbers lie at diverse distances from the AGN, including a range comparable to those we see. For example, de Kool et al. (2001) established that some absorbers in a BALQSO must be $\sim 700$ pc from that nucleus and have kinematic structure sim- 
ilar to the HV components in NGC 1068. High-velocity features are also visible on similar scales in STIS spectra of NGC 4151 (Hutchings et al. 1999), although at more modest velocities $\left(1400 \mathrm{~km} \mathrm{~s}^{-1}\right)$ than we see, and are not clearly connected to massive clouds as in NGC 1068.

\section{FUTURE WORK TO CONSTRAIN FILAMENT PROPERTIES}

The brighter blueshifted knots in NGC 1068 could be mapped in higher ionization lines with UV spectra to establish their optical depths and ionization structure. But this study would be arduous with STIS because of their relative faintness and large reddening. Unfortunately, the complex background emission makes the knots unsuitable targets for the high-efficiency Cosmic Origins Spectrograph (COS) to replace STIS on HST.

Now that specific targets are known up to $2^{\prime \prime}$ from the nucleus, practical spectroscopy is possible with optical and nearIR lines using integral-field spectrometers that are appearing on $8-10 \mathrm{~m}$ telescopes. An interesting constraint follows if such spectra find evidence of the $\mathrm{H}$ I/II recombination front present in optically thick clouds (Shields, Ferland, \& Peterson 1995; Viegas-Aldovandi \& Contini 1989, for example). Constraining the column will better constrain photoionization models to predict the full mass - hence KE, momentum, and ultimately the origin — of each knot and cloud.

\section{SUMMARY}

Our STIS spectral maps have resolved the spatio-kinematic structure of the NLR in NGC 1068, and reveal that many compact knots in the FOC image have large, exclusively blue- shifted velocities, ranging up to $\sim 3200 \mathrm{~km} \mathrm{~s}^{-1}$ from systemic. If these knots are optically thick in the UV continuum, they are good candidates for "associated absorber" clouds present in other AGN. Lying 70-150 pc from the nucleus, several form a broken spatio-kinematic ring of diameter $35 \mathrm{pc}$ that is expanding at up to $1500 \mathrm{~km} \mathrm{~s}^{-1}$. The ring is adjacent to the more massive but slower moving NLR clouds G-K, so may originate from ablata streams from disintegrating molecular clouds that are being photoionized and accelerated radiatively by the AGN or mechanically by a knot in the radio jet.

By resolving this NLR, we have demonstrated that groundbased integral-field spectrometers will be able to constrain the properties of the emitting gas in NGC 1068 by using classical plasma diagnostics in the optical and near-IR. More fundamentally, we may have a convenient view onto gas associated with an important component of AGN that is hard to study elsewhere because it is usually seen in absorption.

This work is based on observations made with the NASA Hubble Space Telescope, obtained at the Space Telescope Science Institute, which is operated by the Association of Universities for Research in Astronomy, Inc., under NASA contract NAS5-26555. These observations are associated with proposal ID GO-7353. We thank Alessandro Capetti for the HST FOC mosaic of NGC 1068, and NASA for support through grants NAG 81027 and GO-7353. GC thanks the Research School of Astronomy and Astrophysics in the Institute for Advanced Studies of the Australian National University, and its Director Prof. J. Mould, for financial support and a stimulating work environment to begin this paper.

\section{REFERENCES}

Alloin, D. et al. 1983, ApJ, 275, 493

Arribas, S., Mediavilla, E., \& Garcia-Lorenzo. B. 1996, ApJ, 463, 509

Axon, D. J., Marconi, A., Macchetto, F. D., Capetti, A., \& Robinson, A. 1998, Ap\&SS, 248, 69

Baldwin, J., Wilson, A. S., \& Whittle, M. 1987, ApJ, 319, 84

Behar, E. et al. 2001, in Mass Outflow in Active Galactic Nuclei: New Perspectives, ASP Conference Series, Vol ?, D. M. Crenshaw, S. B. Kraemer, \& I. M. George (eds.), in press (astro-ph/0106398)

Bicknell, G., Dopita, M. A., Tsvetanov, Z. I., \& Sutherland, R. S. 1998, ApJ, 495,680

Binette, L., Wilson, A. S. \& Storchi-Bergmann, T. 1996, A\&A, 312, 265

Blietz, M. et al. 1994, ApJ, 421, 92

Brinks, E., Skillman, E., Terlevich, R. J., \& Terlevich, E. 1997, Ap\&SS, 248, 23

Caganoff, S. et al. 1991, ApJ, 377, L9

Capetti, A., Macchetto, F. D., \& Lattanzi, M. G. 1997, Ap\&SS, 248, 245

Cecil, G., Bland, J., \& Tully, R. B. 1990, ApJ, 355, 70 (CBT)

Crenshaw, D. M. \& Kraemer, S. B. 2000, ApJ, 532, 247 (CKa)

Crenshaw, D. M. \& Kraemer, S. B. 2000, ApJ, 532, L101 (CKb)

de Kool, M. et al. 2001, ApJ, 548, 609

Dopita, M. A. 1997, Ap\&SS, 248, 93

Dopita, M. A., Groves, B. A., Sutherland, R. S., Binette, L., \& Cecil, G., 2001 submitted to ApJ

Evans, I. et al. 1991, ApJ, 369, L27

Gallimore, J., et al 1996, ApJ, 464, 198 (G96)

Gallimore, J. et al. 1994, ApJ, 422, L13

Groves, B. et al. 2001, in preparation

Hamann, F., Barlow, T. A., Junkkarinen, V., \& Burbidge, E. M. 1997, ApJ, 478, 80

Hutchings, J. et al. 1999, AJ, 118, 2101
Kinkhabwala, A. et al. 2001, BAAS, 33, 812

Kraemer, S. B. \& Crenshaw, D. M. 2000, ApJ, 544, 763 (KC1)

Kraemer, S. B., Ruiz, J. R., \& Crenshaw, D. M. 1998, ApJ, 508, 232

Macchetto, D., Capetti, A., Sparks, W. B., Axon D. J., \& Boksenberg, A. 1994, ApJ, 435, 15

Marconi, A., van der Werf, P. P., Moorwood, A. F. M., \& Oliva, E. 1996, A\&A, 315,335

Nelson, C. H. et al. 2000, ApJ, 531, 257

Ogle, P., Canizares, C. R., Marshall, H. L., Lee, J. C., \& Dewey, D. 2001, in Mass Outflow in Active Galactic Nuclei: New Perspectives, ASP Conference Series, Vol ?, D. M. Crenshaw, S. B. Kraemer, \& I. M. George (eds.), in press

Oliva, E. et al. 2001, A\&A, 369, L5

Paerels, F. et al. 2000, BAAS, 32, 1181

Pécontal, E., Ferruit, P., Binette, L., \& Wilson, A. S. 1997, Ap\&SS, 248, 167 (PFBW)

Roy, A. L., Wilson, A. S., Ulvestad, J. S., \& Colbert, E. J. M. 2001, (astroph/0009408), in Proceedings of the 5th EVN Symposium, ed. J. E. Conway (in press)

Schiano, A. V. R., Christiansen, W. A., \& Knerr, J. M. 1995, ApJ, 439, 237

Shields, J. C., Ferland, G. J., \& Peterson, B. M. 1995, ApJ, 441, 507

Solomon, P. M. et al. 1987, ApJ, 319, 730

Taylor, J., Dyson, J. E., \& Axon, D. J. 1992, MNRAS, 255, 351

Tecza, M., Thatte, N., \& Maiolino, R. 2001, in Galaxies and Their Constituents at the Highest Angular Resolution, IAU Symposium Vol. 205, eds. R. T. Schilizzi, S. Vogel, F. Paresce, \& M. Elvis

Viegas-Aldorovandi, S. \& Contini, A. 1989, A\&A, 215, 253

Walker, M. 1968, ApJ, 151, 71

Wilson, A. S. \& Ulvestad, J. 1987, ApJ, 319, 105

Young, A. J., Wilson, A. S., \& Shopbell, P. L. 2001, ApJ, 556, 6 


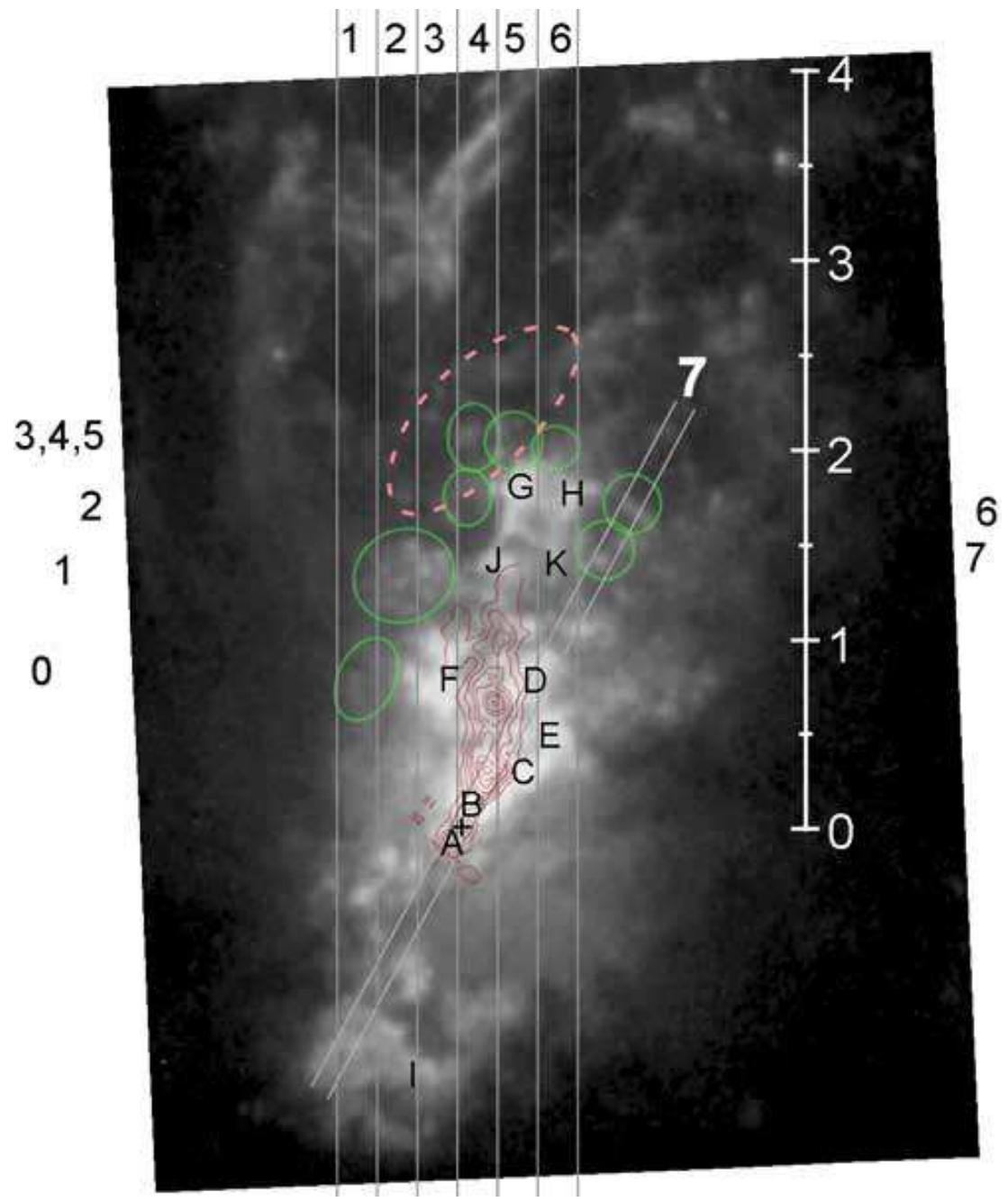

FIG. 1.- FOC image of [O III] $\lambda \lambda 4959,5007$ line emission across the circumnuclear region of NGC 1068 (Macchetto et al. 1994). The greyscale is proportional to the $\log$ of the intensity. The vertical direction is in P.A. $38^{\circ}$, with NE at top. denote the high-velocity regions (HV0-HV7) described in $\$ 3.1 .2$; they are also drawn in Fig. 2 to define the relationship between this image and the locations of the slits. The numbers $0-7$ of these clouds are given outside the figure at left and right. The vertical scale (white line and vertical numbers) originates at the (obscured) nucleus (which coincides with radio source S1, denoted by + ) and is in arcseconds. The numbers at top and the white 7 in the figure refer to the slits, the boundaries of which are shown as vertical and diagonal lines. The spectra from these 7 slit positions are shown in Fig. 2. The location of the Doppler ellipsoid is dashed in magenta. The V-shaped feature discussed in $\$ 3.1 .4$ and $\S 4.3 .2$ is at top, between 3 and $4 "$ radius. 

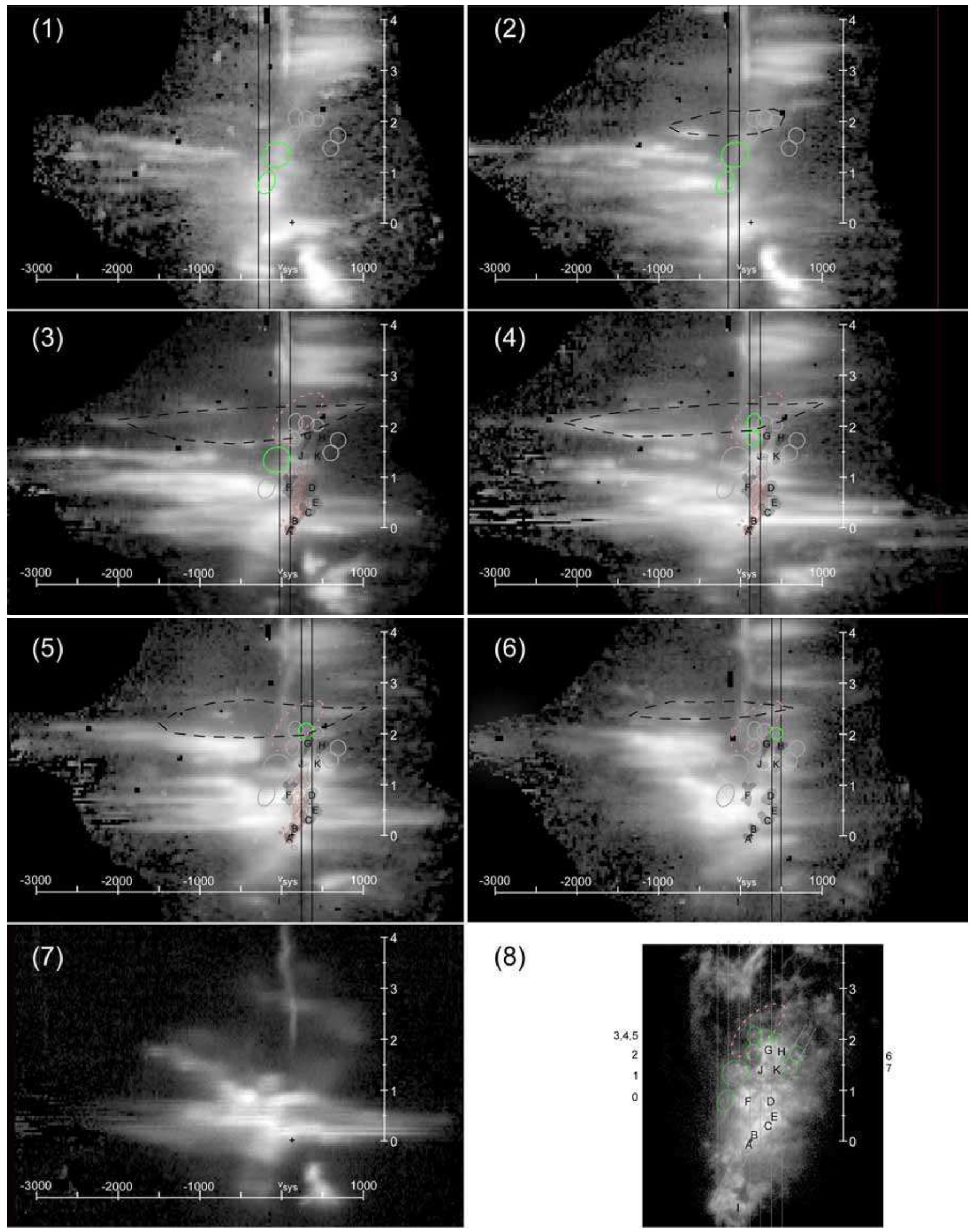

(8)

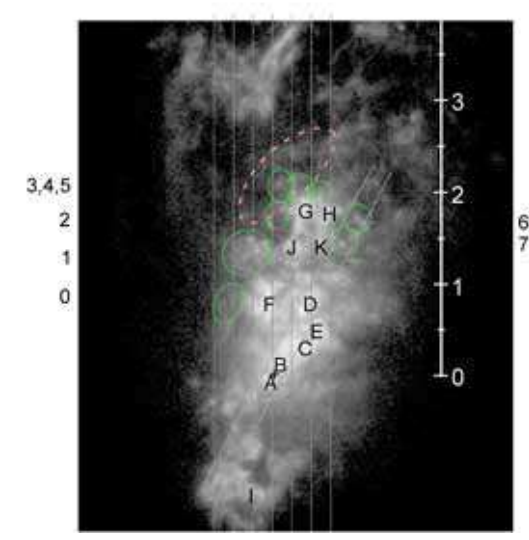

FIG. 2.- Continuum-subtracted, log-scaled intensity, spatial-spectral images of [O III $] \lambda 5007$ emission-line profiles, with position along the slit vertical and wavelength horizontal. The $\lambda 4959$ profile has been subtracted. Slit P.A. is $38^{\circ}$, except for panel (7) where it is P.A. $10^{\circ}$ (marked by the parallel lines in panel 8 , which repeats the FOC image shown in Fig. 1). The archival spectrum in panel (7) has been binned spatially to resemble spectra in panels (1-6). The position of the slit w.r.t. the circles and ellipses denoting the high-velocity (HV) complexes (see Fig. 1 and Table 2) is shown in panels (1-6). The vertical scale is in arc-seconds from the nucleus (radio source S1) and the horizontal scale is in $\mathrm{km} \mathrm{s}^{-1}$ relative to the galaxy systemic value. Note the very broad $\left(>5,000 \mathrm{~km} \mathrm{~s}{ }^{-1} \mathrm{FWZI}\right.$ ) [O III] profiles at knot B in slit 4. The Doppler ellipsoid (\$4.3.3), which is dashed in magenta in Fig. 1, has its kinematic manifestation marked by a black dashed oval in panels 2-6. A high-fidelity, color version of this figure is at http://www.thececils.org/science/n1068/spectra.jpg 

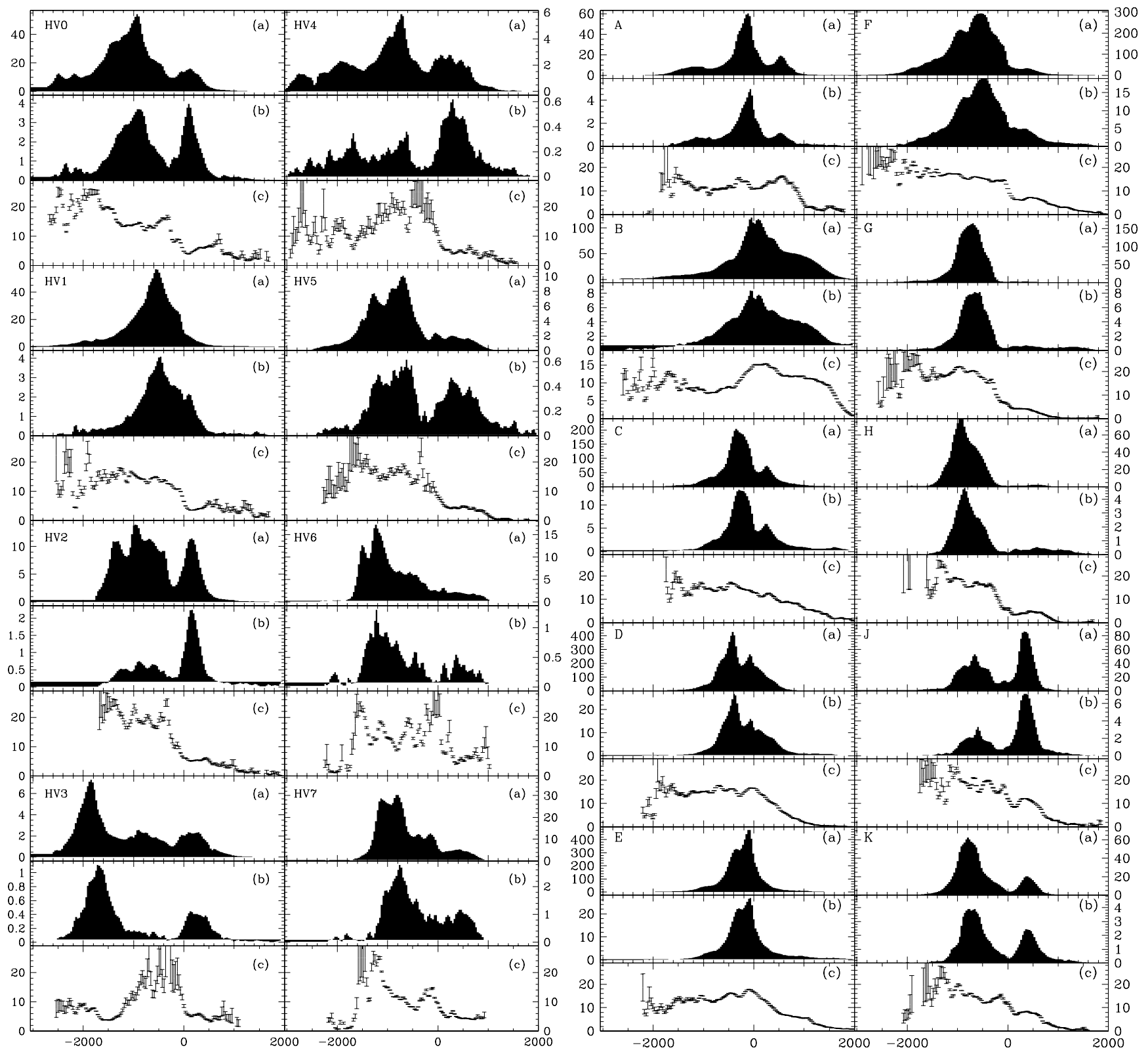

FIG. 3.- Shown are the dereddened line profiles of the high-velocity knots (HV0-HV7: left diagram) and more massive clouds (A-K: right diagram). In each set of three spectra, the top profile (a) is [O III] $\lambda 5007$ after contamination by $\lambda 4959$ has been removed, the middle (b) is $\mathrm{H} \beta$, and the bottom (c) shows the range of the ratio $[\mathrm{O}$ III $] \lambda 5007 / \mathrm{H} \beta$ at each velocity within $\pm 1 \sigma$ statistical plus estimated systematic uncertainties from the continuum subtraction. Velocities are relative to the galaxy systemic value. The spectral resolution is $60 \mathrm{~km} \mathrm{~s}^{-1} \mathrm{FWHM}$ for the [O III] and $\mathrm{H} \beta$ profiles; the line ratio plots have been smoothed with a boxcar of width $160 \mathrm{~km} \mathrm{~s}^{-1}$. The vertical scale is in units of $10^{-18} \mathrm{ergs} \mathrm{s}^{-1} \mathrm{~cm}^{-2}$ pixel $^{-1}$, where the pixel bin width is $33 \mathrm{~km} \mathrm{~s}^{-1}$. 


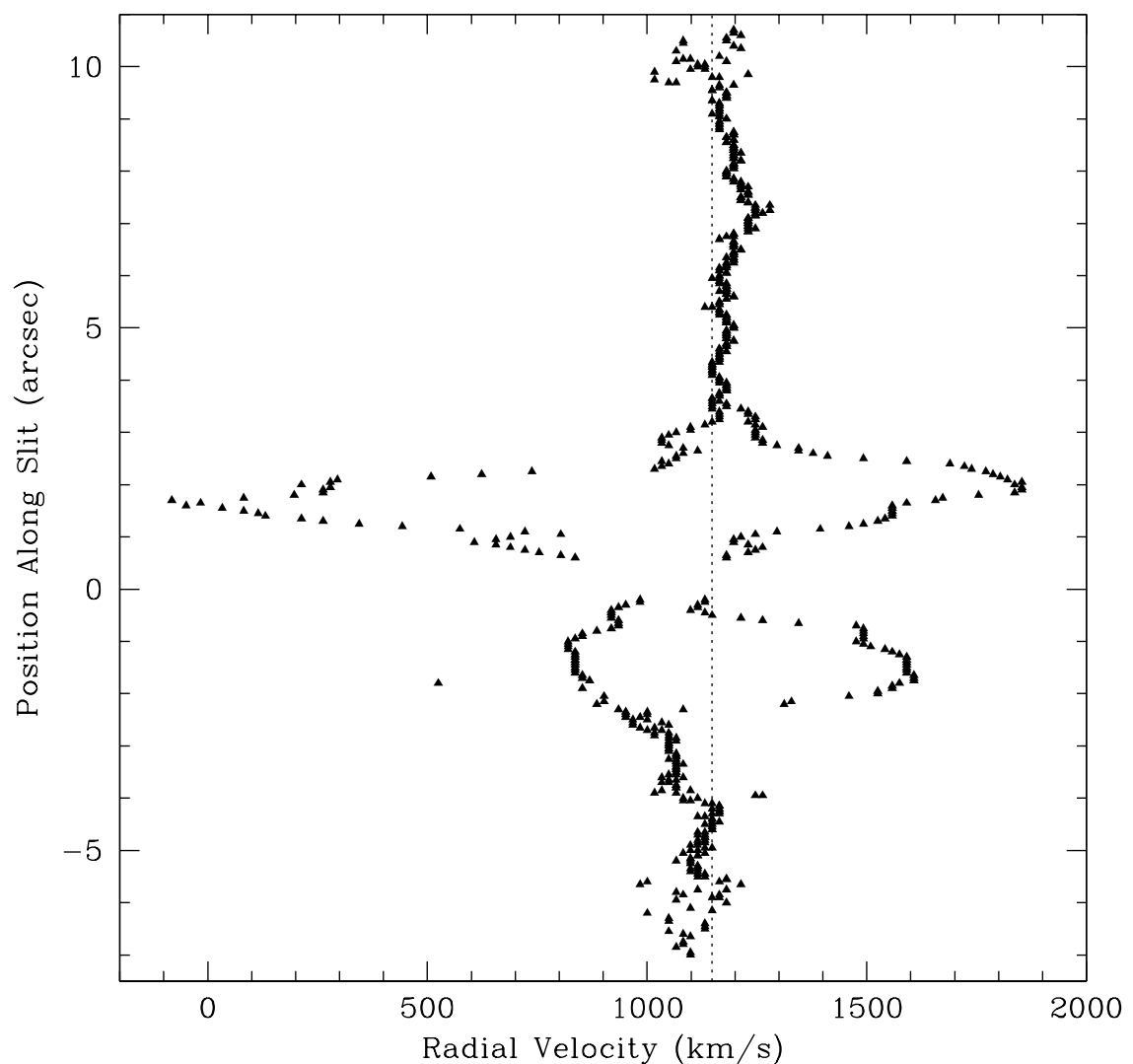

FIG. 4.- Velocities of the flux centroids of the two main emission-line components in each continuum-subtracted line profile as a function of distance from the nucleus, obtained from an archival M-grating spectrum (slit P.A. $=10^{\circ}$ ). NE is at top and SW is at bottom. The vertical dotted line shows the systemic velocity of the galaxy. The velocity field is similar to that shown in Fig. 2 of CKb that was extracted along a slightly different P.A. The high-velocity, blueshifted motions between 1." $5-2$." 5 radius NE of the nucleus result from knots 6 and 7.

TABLE 1

STIS LONG-SLIT OBSERVATIONS OF NGC 1068

\begin{tabular}{|c|c|c|c|c|c|c|}
\hline Data Set & Date & Grating & $\begin{array}{l}\text { Dispersion } \\
\left(\AA \text { pixel }^{-1}\right)\end{array}$ & $\begin{array}{l}\text { Spectral } \\
\text { Range }\end{array}$ & $\begin{array}{c}\text { Exposure } \\
\text { (s) }\end{array}$ & $\begin{array}{c}\text { Slit P.A. } \\
\text { (deg.) }\end{array}$ \\
\hline o56502010 & 1999 Oct 2 & G430M & 0.6 & $4818-5104$ & 2585 & 38 \\
\hline o56502020 & 1999 Oct 2 & G430M & 0.6 & $4818-5104$ & 2775 & 38 \\
\hline o56502030 & 1999 Oct 2 & G430M & 0.6 & $4818-5104$ & 2775 & 38 \\
\hline o56502040 & 1999 Oct 2 & G430M & 0.6 & $4818-5104$ & 2775 & 38 \\
\hline o56502050 & 1999 Oct 2 & G430M & 0.6 & $4818-5104$ & 2772 & 38 \\
\hline o56503010 & 2000 Sept 22 & G430M & 0.6 & $4818-5104$ & 2294 & 38 \\
\hline o56503020 & 2000 Sept 22 & G430M & 0.6 & $4818-5104$ & 2853 & 38 \\
\hline o56503030 & 2000 Sept 22 & G230L & 1.6 & $1568-3184$ & 2376 & 38 \\
\hline o56503040 & 2000 Sept 22 & G230L & 1.6 & $1568-3184$ & 2376 & 38 \\
\hline o56503050 & 2000 Sept 22 & G230L & 1.6 & $1568-3184$ & 2376 & 38 \\
\hline o56503060 & 2000 Sept 22 & G230L & 1.6 & $1568-3184$ & 2376 & 38 \\
\hline o56501010 & 1999 Sept 22 & G140L & 0.6 & $1140-1730$ & 1425 & 38 \\
\hline o56501020 & 1999 Sept 22 & G140L & 0.6 & $1140-1730$ & 1425 & 38 \\
\hline o56501030 & 1999 Sept 22 & G140L & 0.6 & $1140-1730$ & 1425 & 38 \\
\hline o5lj01050 & 2000 Jan 14 & G430M & 0.3 & $4818-5104$ & 800 & 10 \\
\hline o5lj01060 & 2000 Jan 14 & G430M & 0.3 & $4818-5104$ & 846 & 10 \\
\hline o5lj01070a & 2000 Jan 14 & G430M & 0.3 & $4818-5104$ & 960 & 10 \\
\hline $\mathrm{o} 5 \mathrm{lj} 01080^{\mathrm{a}}$ & 2000 Jan 14 & G430L & 0.6 & $2900-5700$ & 720 & 10 \\
\hline o5lj01090 & 2000 Jan 14 & G430L & 0.6 & $2900-5700$ & 720 & 10 \\
\hline $\mathrm{o} 5 \mathrm{lj} 010 \mathrm{a} 0^{\mathrm{a}}$ & 2000 Jan 14 & G430L & 0.6 & $2900-5700$ & 821 & 10 \\
\hline
\end{tabular}

${ }^{a}$ Archival exposure. 

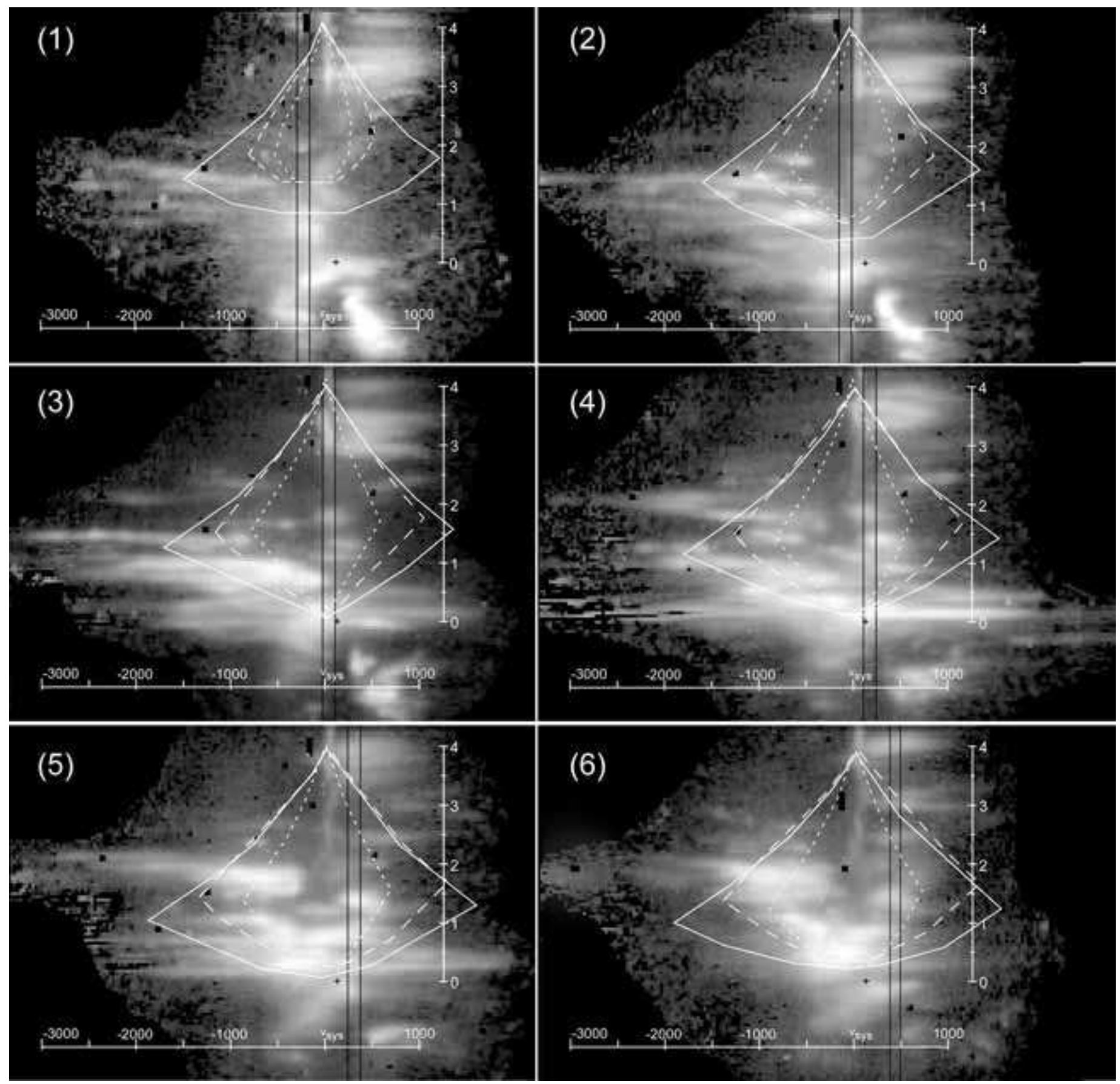

FIG. 5.- Same display format and region as for panels 1-6 in Fig. 2, but now with contours to show the space-velocity projections of the outer cones in the three bi-conical outflow models described in the text: (solid lines) increasing the maximum outflow velocity from the nucleus to $2500 \mathrm{~km} \mathrm{~s}^{-1}$ at $r_{i}$, (long dashed lines) having the gas expand cylindrically from the jet axis at close to the observed velocity, (short dashed lines) outflow model of CKb. At best these models match only the blue wing of the data within $1 . " 5 \mathrm{NE}$ of the nucleus. The red wing is not fit at all. 


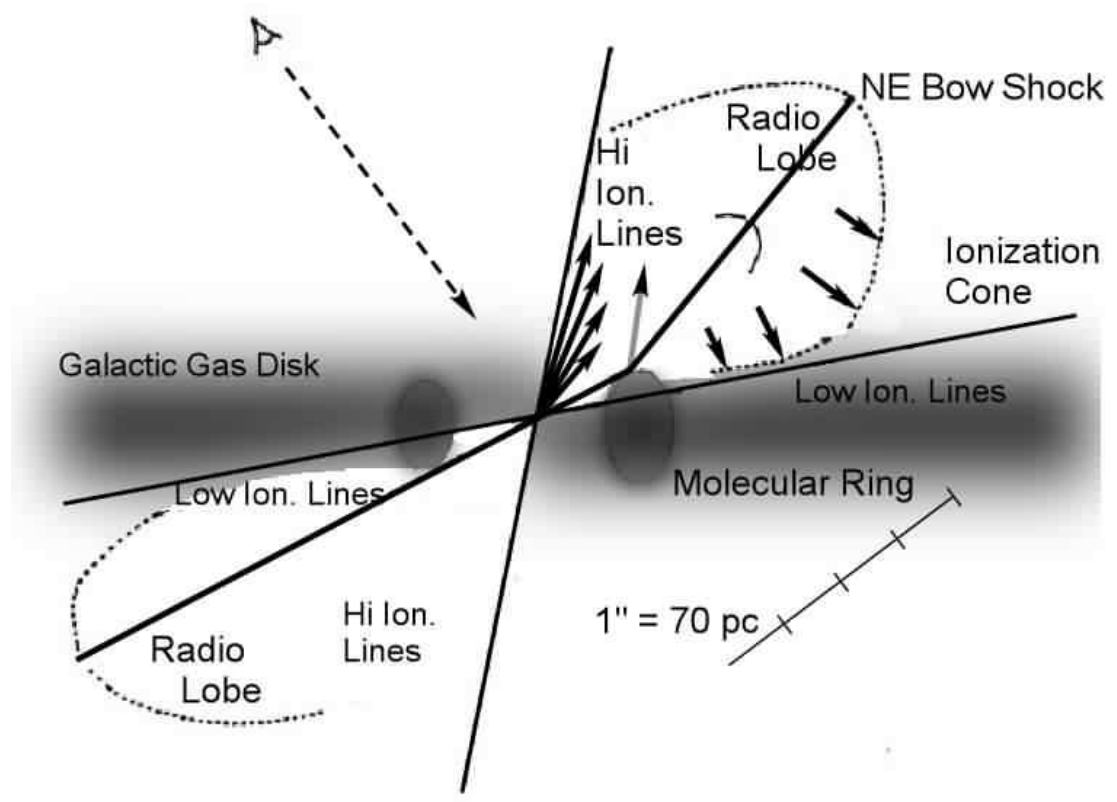

FIG. 6. - Kinematic model of the NLR refined from that proposed by Tecza, Thatte, \& Maiolino (2001). To represent patterns seen in our spectral maps, we have (i) added radial expansion from the nucleus, the velocity of that outflow appearing to increase with its angle of travel above the disk; (ii) removed blueshifted gas associated with the expanding radio lobe because our data show only redshifted gas that is being pushed into the galaxy disk; (iii) added the gray arrow to represent possible ablata from the molecular ring (which may be a highly warped outer extension of the putative torus obscuring our view of the AGN).

TABLE 2

PROPERTIES OF THE NLR CLOUDS IN NGC 1068

\begin{tabular}{|c|c|c|c|c|c|c|}
\hline $\begin{array}{l}\text { Cloud } \\
\text { Name }\end{array}$ & $\begin{array}{c}\text { Area }^{\mathrm{a}} \\
\left(\operatorname{arcsec}^{2}\right)\end{array}$ & $\begin{array}{c}\left.\mathrm{L}_{[\mathrm{O}} \mathrm{III}\right] \lambda 5007^{\mathrm{b}} \\
\left(10^{40} \mathrm{ergs} \mathrm{s}^{-1}\right)\end{array}$ & $\begin{array}{c}\text { Ionized H Mass } \\
\left(10^{2} \mathrm{M}_{\odot} / n_{e, 4}\right)\end{array}$ & $\begin{array}{c}{ }^{\mathrm{c}}[\mathrm{O} \mathrm{III}] \lambda 5007^{\mathrm{d}} \\
/ \mathrm{H} \beta \text { flux }\end{array}$ & $\begin{array}{l}\log \mathrm{KE}^{\mathrm{e}} \\
\left(\mathrm{ergs} / \mathrm{n}_{e, 4}\right)\end{array}$ & $\begin{array}{l}\log \text { momentum } \\
\left(\text { dynes } \mathrm{s} / \mathrm{n}_{e, 4}\right)\end{array}$ \\
\hline A & 0.03 & 3.1 & 16.6 & 12.3 & 51.50 & 43.87 \\
\hline B & 0.04 & 10.8 & 63.0 & 11.5 & 52.33 & 44.61 \\
\hline $\mathrm{C}$ & 0.06 & 10.9 & 55.3 & 13.2 & 51.98 & 44.38 \\
\hline D & 0.04 & 21.1 & 100.8 & 13.9 & 52.18 & 44.64 \\
\hline $\mathrm{E}$ & 0.09 & 20.7 & 96.9 & 14.2 & 52.16 & 44.58 \\
\hline F & 0.06 & 24.5 & 116.2 & 13.9 & 52.58 & 44.88 \\
\hline G & 0.06 & 8.1 & 32.5 & 16.7 & 52.02 & 44.37 \\
\hline $\mathrm{H}$ & 0.06 & 3.7 & 16.6 & 14.7 & 51.75 & 44.11 \\
\hline $\mathrm{J}$ & 0.06 & 3.3 & 17.6 & 12.7 & 51.53 & 43.97 \\
\hline K & 0.06 & 3.5 & 18.8 & 12.4 & 51.65 & 44.07 \\
\hline HVO & $\ldots$ & 3.6 & 20.6 & 10.9 & 51.79 & 44.09 \\
\hline HV1 & $\ldots$ & 5.0 & 26.4 & 11.8 & 52.17 & 44.35 \\
\hline HV2 & $\cdots$ & 1.4 & 8.1 & 11.3 & 51.31 & 43.65 \\
\hline HV3 & $\ldots$ & 0.6 & 5.5 & 7.0 & 51.75 & 43.84 \\
\hline HV4 & $\ldots$ & 0.6 & 4.2 & 9.8 & 51.48 & 43.61 \\
\hline HV5 & $\ldots$ & 0.8 & 5.0 & 11.2 & 51.33 & 43.60 \\
\hline HV6 & $\ldots$ & 1.1 & 6.2 & 12.2 & 51.49 & 43.74 \\
\hline HV7 & $\ldots$ & 2.1 & 13.9 & 10.3 & 51.60 & 43.96 \\
\hline
\end{tabular}

${ }^{a}$ Area of each distinct kinematic component on the sky, typically uncertain by $\pm 0.015 \operatorname{arcsec}^{2}$.

${ }^{\mathrm{b}}$ Line fluxes at velocities below galaxy systemic were dereddened by $\mathrm{E}(\mathrm{B}-\mathrm{V})=0.35$, above systemic by 0.22 .

${ }^{\mathrm{c}}$ Assumes case-B recombination conditions and a pure $\mathrm{H}$ plasma.

${ }^{\mathrm{d}}$ Velocity averaged flux ratio, typically uncertain by \pm 0.3 .

${ }^{\mathrm{e}}$ Minimum values, assuming that all velocity is along the line of sight. Density $\mathrm{n}_{e, 4}$ is in units of $10^{4} \mathrm{~cm}^{-3}$. 